\title{
Image Based Acquisition and Tracking for Multi- Access Laser Communications
}

\author{
Charles W. Hindman, Seth L. Lacy, \& Nicole Hatten \\ Air Force Research Laboratory \\ 3550 Aberdeen Ave SE \\ Kirtland AFB, NM 87117 \\ 505-853-4746 \\ \{Charles.hindman | Seth.Lacy\}@kirtland.af.mil \\ Nicole.Hatten@student.Mercer.edu
}

\begin{abstract}
Long-range laser communications links offer many potential advantages to satellite communications systems including high bandwidth, security, and a small physical footprint. However, the establishment of a laser communications link can be very difficult and time consuming due to the extremely small beam divergences and stringent platform stability requirements inherent to lasercom systems. Most of the difficulty lies in the first step of actually painting the partner terminal with a laser spot or detecting said spot due to large uncertainties, terminal vibrations and jitter, bright optical backgrounds and clutter. A detailed physical and statistical model of this first detection process has been developed and verified against computer simulations using representative background images to ensure the success of this critical first step in the acquisition process, enabling future designers to have some confidence that their terminals will be able to acquire ${ }^{12}$.
\end{abstract}

\section{TABLE OF CONTENTS}

1. INTRODUCTION 1

2. FPA MODELING .......................................................... 1

3. JITTER MODELING .......................................................... 3

4. DETECTION STATISTICS................................................... 3

5. IMAge SYNTHESIS ANd PROCESSING ............................... 4

6. EXAMPLE AND SIMULATION RESULTS ......................... 5

7. CONCLUSIONS ............................................................ 9

REFERENCES .................................................................... 9

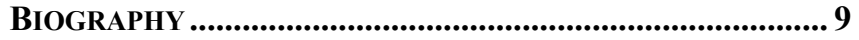

\section{INTRODUCTION}

Future space-based communications systems are investigating the use of laser-based systems due to the revolutionary increases in communications bandwidth, security, and SWaP enabled by the use of laser light as the information carrier. Several current and future space-based systems, such as the ESA's Artemis, the JAXA's OICETs and the DOD's TSAT [1-6], are investigating or have demonstrated lasercom links between a satellite and another satellite or a high-flying aircraft.

\footnotetext{
${ }^{1}$ U.S. Government work not protected by U.S. copyright

${ }^{2} 1596 / 3$
}

Establishing a lasercom link between two terminals requires an acquisition process to enable the terminals to find and lock onto each other with very small divergence beams (1 to $100 \mu \mathrm{rads}$, depending on range and aperture sizes). The initial step of this acquisition process involves each terminal scanning an illumination beam towards its partner terminal while trying to detect and discern the incoming optical signal in the presence of platform vibrations and cluttered optical backgrounds. This initial step of scan and detect can be the most difficult and time consuming step in the process of setting up a lasercom link, especially when the system is plagued by false alarms or missed detections.

Early free-space laser communications systems typically used a quad-cell detector for both acquisition and tracking $[7,8]$, possibly utilizing a modulated signal to cut down on the large background noise inherent in such a system with the field of view required to encompass the uncertainty in the location of the far terminal. As sensor technology has evolved, designers have been drawn towards utilizing CCDtype focal plane arrays (FPAs) because of their excellent responsivity, low noise characteristics, and the smaller background signals created by segmenting the field of view into hundreds to millions of pixels.

The remainder of the paper is organized as follows: First, we present a model of a FPA that includes the relevant noise sources. Next, a simplified model to represent spacecraft jitter is presented. Then the statistical basis underlying the first detection process is developed, and after that the image processing steps to achieve first detection are developed. Finally, the example background image and simulation results are presented, followed by the conclusion.

\section{FPA MODELING}

The focal plane array devices likely to be used in laser communications systems are $\mathrm{Si}$, InGaAs or other types of arrays that act as power integrators, converting the incoming photons into electrons and then counting the number of electrons detected. The principles of operation and typical noise sources for these devices are well understood due to their extensive use in astronomical application (see for example [9]). The number of electrons produced for a given 
energy input onto the FPA can be determined by the following equation:

$$
N_{p e}=s_{i n} \cdot Q E \cdot \frac{\lambda}{\hbar c}
$$

where:

$\mathrm{N}_{\mathrm{pe}}=$ Number of photoelectrons produced

$\mathrm{S}_{\mathrm{in}}=$ Energy input in 1 integration period

$\mathrm{QE}=$ FPA quantum efficiency

$\lambda=$ Wavelength

$\hbar=$ Planck's constant (6.626E-34 Js)

$\mathrm{c}=$ Speed of light $(2.99792458 \mathrm{E} 8 \mathrm{~m} / \mathrm{s})$

The electrons are then read out by being digitized into $\mathrm{N}$ bits, perhaps after an amplification stage. The read out from the FPA then becomes:

$$
R=\frac{N_{p e}}{\text { fullwell } / 2^{n}}
$$

Where:

$\mathrm{R}=$ Readout from the FPA

Fullwell $=$ saturation level of a FPA pixel, in electrons

$\mathrm{n}=$ Number of bits in digitizer

Several sources of noise conspire to make life more difficult. Typical noise sources are described in detail below and include shot noise, read out noise, dark current noise, background clutter noise, digitization noise, and pixel non-uniformity noise.

Shot noise is a Poisson process that arises from the quantum mechanical nature of photon arrival counting, and can be represented as:

$$
N_{\text {shot }}=\sqrt{\text { signal }+ \text { background }+ \text { dark }}
$$

Where:

Signal $=\#$ of signal electons

Background $=\#$ of background image electrons

Dark $=\#$ of dark current electrons

Read-out noise is associated with the process of reading out the electron well. It can be rate dependent, but is considered to be independent of rate in this simulation. The value is device dependent, but values of tens to a few thousand electrons are typical.

Dark current noise is caused by the thermal energy of the FPA. It becomes important as the wavelength is increased and integration times grow longer. For the wavelengths and short integration times inherent to lasercom systems, it can be assumed to be negligible.

Background clutter noise is the residual image that is left over after the background has been subtracted from the image, and can be considered as arising from three sources: errors in registering the background image; motion of the optical line of sight with respect to the background; and changes in the background image itself. It can be modeled as some percentage of the background signal, where $\mathrm{cf}$ is the clutter factor:

$$
N_{b c}=c f * \text { background }
$$

Digitization noise arises from binning the electron counts in the analog to digital converter, and is given by:

$$
N_{a d}=\frac{\text { FullWell }}{2^{n+1}}
$$

Pixel non-uniformity noise arises from the fixed pattern pixel-to-pixel variations in responsivity. This can be quite large initially; however, careful calibration with a flat field can reduce it to a small value. Unfortunately, the spatial nonuniformity is not constant and can drift slowly in time (see [16]). The noise term can be given in terms of a nonuniformity factor $\mathrm{n}_{\mathrm{u}}$ :

$$
N_{n u}=\left(n_{u} *(\text { signal }+ \text { background }+ \text { dark })\right)
$$

The total noise per pixel is then given by:

$$
\sigma_{n}=\sqrt{N_{\text {shot }}^{2}+N_{\text {read }}^{2}+N_{b c}^{2}+N_{a d}^{2}+N_{n u}^{2}}
$$

Computing the SNR for the centroiding window requires summing up the signal and noise terms in each pixel, and is given by:

$$
S N R=\frac{\sum_{i, j} S_{i, j}}{\sqrt{\sum_{i, j} N_{i, j}}}
$$

An estimate of the Noise Equivalent Angle (NEA) is proportional to the inverse of the SNR:

$$
N E A=\frac{\eta}{S N R} \text { ifov }
$$

where $\eta$ is a dimensionless conversion factor. Ortiz, Lee and Hemmati [10] give $\eta$ to be:

$$
\eta=\sqrt{\frac{N_{o}\left(N_{o}+1\right)}{3}}
$$

where $\mathrm{N}_{\mathrm{o}}$ is the truncated half width of the window.; but we believe this may be a misprint and propose an estimation of $\eta$ as:

$$
\eta=\sqrt{\frac{3}{N_{p}\left(N_{p}+1\right)}}
$$

where $\mathrm{N}_{\mathrm{p}}$ is the window size; However, results from our simulations show this to be a very good estimator only for some combinations of window size and SNR; in other cases it can be off by up to a factor of 3 . This form of the NEA equation illustrates the trade that must be made to determine the window size: as $\mathrm{N}_{\mathrm{p}}$ goes up, $\eta$ decreases, leading to a smaller NEA, but the SNR also drops with increasing $\mathrm{N}_{\mathrm{p}}$, 
which leads to a larger NEA. The optimal window size can be determined based on an analysis of the expected signal and noise terms

\section{JitTer Modeling}

Optical communications payloads are typically secondary payloads on larger spacecraft and are subject to the jitter vibration environment caused by other onboard devices such as momentum wheels, solar arrays, thrusters, etc. In the design process, the actual or expected vibration spectrum for the platform the lasercom payload is destined for should be used. Unfortunately, only a few data points containing satellite rotational vibration data are available [11,12]. For the purposes of this paper, a typical vibration environment from [7] was assumed, with the PSD shown in the top half of Figure 1:
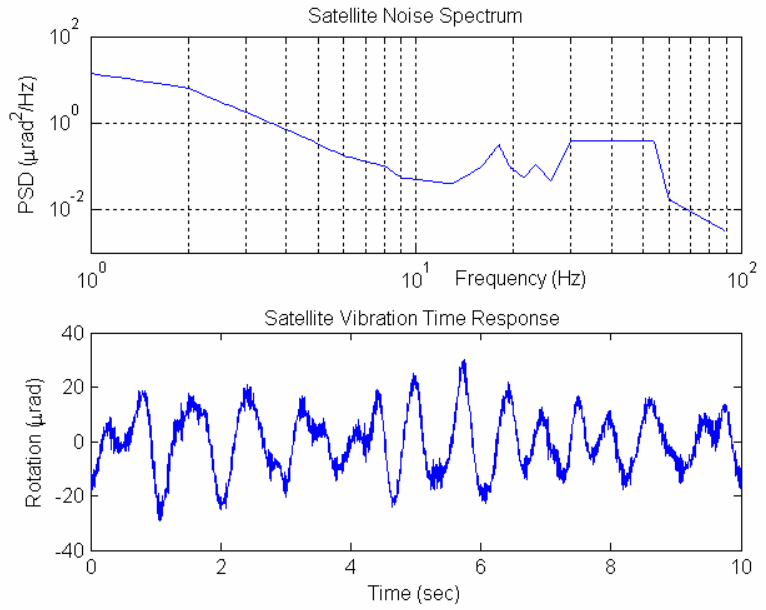

Figure 1: Jitter PSD (1-axis) and Time History

A time history was created from this PSD by applying an appropriately weighted inverse Fourier transform to the PSD, with a random phase assigned to each frequency, as shown in the bottom half of Figure 1. Both $\mathrm{X}$ and $\mathrm{Y}$ time histories were created, and were uncorrelated when used in the simulations later in the paper.

\section{DETECTION STATISTICS}

Simple detection theory as described in $[13,14]$ is used to build up the detection statistics for the entire FPA. The basis for this is shown in Figure 2 and depends on the PDFs for the noise and the signal $\left(\mathrm{P}_{\mathrm{n}}\right.$ and $\left.\mathrm{P}_{\mathrm{n}+\mathrm{s}}\right)$, the probability of false alarm $\left(\mathrm{p}_{\mathrm{fa}}\right)$, the probability of detection $\left(\mathrm{p}_{\mathrm{d}}\right)$ and the threshold level (th). For a given noise PDF, setting the threshold level determines the probability of false alarm by:

$$
p_{f a}=\int_{t h}^{\infty} P_{n}(x) d x
$$

Similarly, the probability of detection is determined by:

$$
p_{d}=\int_{t h}^{\infty} P_{n+s}(x) d x
$$

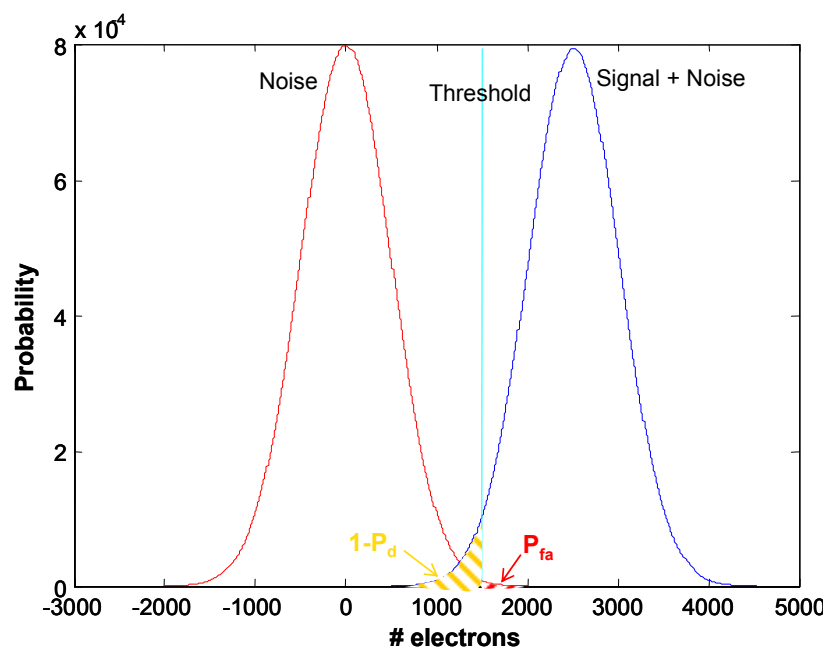

Figure 2: Detection Statistics

Equations $(12,13)$ are for a single detector at a single point in time (designated as $\mathrm{P}_{\text {fa-p }}$, probability of false alarm per pixel). When using a FPA as a detector, the designer is typically given a higher level system specification such as "a $1 \%$ chance of a false alarm per minute" $\left(\mathrm{P}_{\text {fa-s }}\right.$, probability of false alarm, system), which requires computing the false alarm rate over all pixels and a number of frames. The required probability of false alarm per frame $\left(\mathrm{P}_{\mathrm{fa}-\mathrm{f}}\right)$ can be derived from the system probability by:

$$
P_{f a-f}=\frac{P_{f a-s}}{F R \cdot T}
$$

where FR is the frame rate and $\mathrm{T}$ the time interval for the specification (i.e., one minute). Computing the required pixel false alarm rate from the frame false alarm rate is accomplished by:

$$
P_{f a-f}=1-\left(1-P_{f a-p}\right)^{N_{p}}
$$

Where $N_{p}$ is the number of pixels per frame, $N^{2}$. This assumes a false alarm is declared for a given frame even if a true detection occurs in some other pixel in the frame. Combining Equations $(14,15)$ and solving for $\mathrm{P}_{\text {fa-p }}$ gives:

$$
P_{f a-p}=1-\left(1-\frac{P_{f a-s}}{F R \cdot T}\right)^{1 / N_{p}}
$$

As the specification for the required false alarm rate becomes more restrictive, the required threshold setting will move to the right in Figure 2. The requirement for the probability of detection $\left(\mathrm{P}_{\mathrm{d}}\right)$, coupled with the level the threshold is set at, determines how far to the right the PDF $\mathrm{P}_{\mathrm{n}+\mathrm{s}}$ must shift, which is realized by setting a requirement on the received signal to noise ratio (SNR). 
Most systems will have a limit to the achievable SNR, especially in the beginning stage of acquisition, which by the above analysis sets a limit on the false alarm rate that can be realized for any given sensor. Fortunately, there are other design options involving multiple pixels, frames, and signal patterns that can be exploited to reduce the required SNR while still meeting requirements for $P_{f a}$ and $P_{d}$. For example, if the signal is required to be present for $\mathrm{n}_{\mathrm{f}}$ frames (consecutive or in some predetermined sequence), Equation (15) becomes:

$$
P_{f a-f}=1-\left(1-P_{f a-p}^{n_{f}}\right)^{N_{p}}
$$

Alternatively, you can utilize the optical design of your sensor to create a point spread function that will encompass multiple pixels. Of course, in this case the trade is a lower SNR in each pixel. However, you are likely to require the PSF to span multiple pixels in any case for centroiding accuracy reasons. For $\mathrm{n}_{\mathrm{c}}$ contiguous pixels, Equation (15) becomes:

$$
P_{f a-f}=1-\left(1-P_{f a-p}^{n_{c}}\right)^{N_{p n_{c}}}
$$

In this case, the number of pixels $\mathrm{N}_{\mathrm{p}}$ is replaced by the number of $\mathrm{n}_{\mathrm{c}}$ contiguous pixel groupings $\mathrm{N}_{\text {pnc }}$. For $\mathrm{n}_{\mathrm{c}}=2$ :

$$
N_{p n_{c}}=4 N^{2}-6 N+2
$$

To illustrate the advantage schemes such as these provide, the following situation is analyzed:

$$
\begin{aligned}
& \mathrm{N}=512 \text { (number of pixels in } 1 \text { direction) } \\
& \mathrm{FR}=60 \mathrm{~Hz} \text { (frame rate of camera in acquisition } \\
& \text { mode) } \\
& \mathrm{T}=180 \text { seconds (time over which acquisition is } \\
& \quad \text { attempted) } \\
& \mathrm{P}_{\text {fa-s }}=1 \% \text { (required system-level false alarm } \\
& \text { probability) }
\end{aligned}
$$

Using these parameters, the values in

Table 1 were generated for $n_{c}=1,2,3$ and $n_{f}=1,2,3$. It is apparent that vast reductions in the required false alarm rate (and hence, SNR) can be achieved by these techniques.

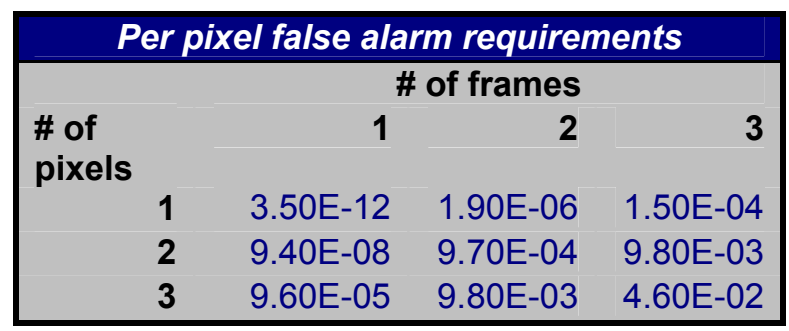

Table 1: Derived False Alarm Rates, Per Pixel

\section{Image Synthesis And Processing}

Several image processing steps are required to go from the input image to a time sequence of noisy, jittering images, and then to perform the background suppression and acquisition detection functions. For the purpose of the acquisition simulation developed here, the input image is assumed to be a 'truth' image, devoid of any noise effects. Jitter and noise are then added to the input image to produce a sequence of input images, and a laser spot with the desired characteristics is synthesized and added to the sequence at a random time stamp. Finally, the image sequence is analyzed to detect an acquisition by using background suppression and the detection algorithms discussed in the previous section.

Jitter is added to the image sequence by taking the jitter time histories as developed in Section 3. The jitter files are first converted from microradians to pixels by dividing by the pixel's instantaneous field of view (iFOV). A several pixel wide buffer zone is removed from the edges of the input image to allow for translation and drift. If the original image is denoted as $\mathrm{A}$, and a translation of $(\mathrm{X}, \mathrm{Y})$ whole pixels is denoted as $A_{x, y}$, while a translation of partial pixels is denoted as $A_{i, j}$. To produce the image that is shifted to $A_{x+i, y+j}$, the image is first shifted by $(x, y)$ pixels and then appropriately interpolated with the image shifted to $A_{\operatorname{ciel}(x+I), \operatorname{ciel}(y+j)}$.

The more difficult task is to add in noise to each frame. Each shifted frame is used as a basis for adding in per pixel noise based on the noise processes described in Section 2. The standard deviation of the noise $\sigma_{\mathrm{n}}$ from Equation (7) is used to randomly generate a new pixel value from Equation (1) as follows:

$$
N_{p e}(k)=v(k)
$$

Where:

$$
E[v(k)]=s_{i n} \cdot Q E \cdot \frac{\lambda}{\hbar c}, \quad \operatorname{var}[v(k)]=\sigma_{n}^{2}
$$

Once a sequence of noisy, jittered images has been generated, a simulated laser spot is generated using an Airy profile as given by:

$$
I(\theta)=I_{o}\left(\frac{2 J_{1}\left(\frac{\pi \theta D_{e f f}}{\lambda}\right)}{\left(\frac{\pi \theta D_{e f f}}{\lambda}\right)}\right)^{2}
$$

With:

$$
I_{o}=\frac{P \pi D_{e f f}^{2}}{4 \lambda^{2}}
$$

Where $\mathrm{P}$ is the input power and $\mathrm{D}_{\text {eff }}$ determines the radius of the Airy spot. The spot is randomly located within the array at the point $\left(\mathrm{C}_{\mathrm{x}}, \mathrm{C}_{\mathrm{y}}\right)$ and an integration is performed over the boundaries of each pixel as follows: 


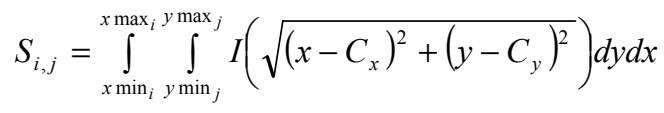

The sequence of frames is analyzed by first removing the strong background signal from the images. Several techniques were analyzed, depending on which detection algorithm was chosen, but all are of the form:

$$
B^{t}=A^{t}-A^{t-m}
$$

Detection is then simply based on threshold setting and applying the specified algorithm (ie, 2 consecutive frames or 2 contiguous pixels).

\section{EXAMPLE AND SimULATION RESULTS}

An example image was used as a basis for a simulation of this process from the Enhanced Thematic Mapper Plus $(\mathrm{ETM}+)$ camera on Landsat 7 [15]. This camera encompasses seven spectral bands, ranging from .45 micrometers to 2.35 micrometers; an image from band 2 (.52 to .6 $\mu$ ) was chosen. Also, for this satellite, the Instantaneous Field of View (IFOV) equals 42.5 microradians equating to a nominal ground resolution of 30 meters. Landsat 7 satellite has a $40.64 \mathrm{~cm}$ aperture size with a $243.8 \mathrm{~cm}$ focal length. The example image is shown in Figure 3:

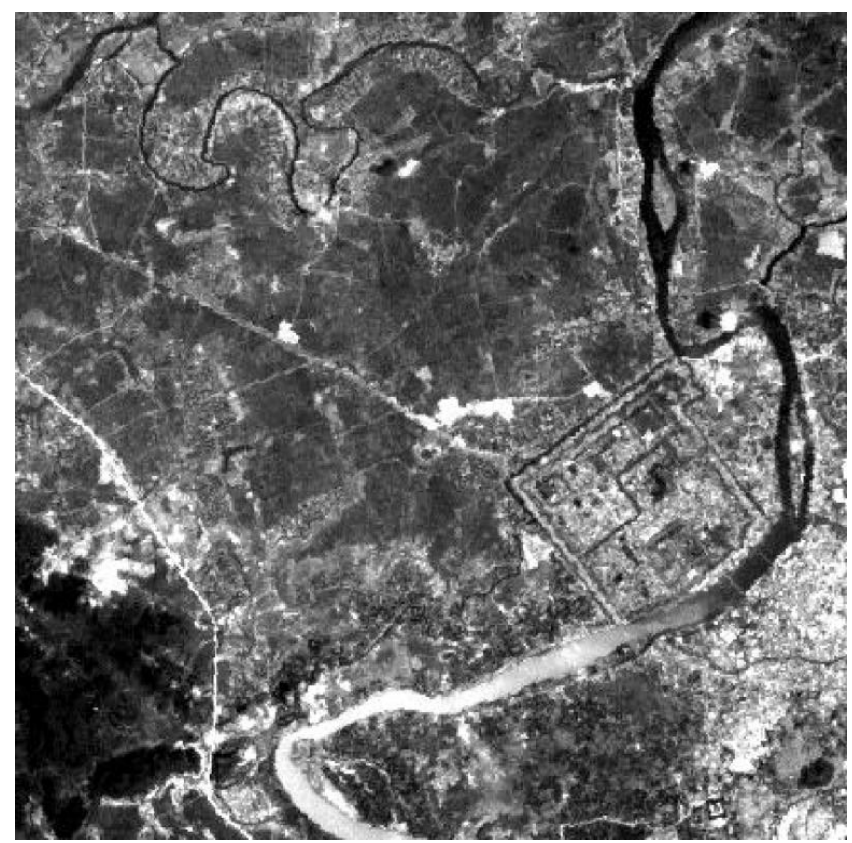

Figure 3: Example Reference Image

This image has been optimized for imaging purposes, as the histogram shown in Figure 4 clearly shows (the background brightness values cover the full range of the camera). A sensor with parameters optimized to be a lasercom detector might produce images that look much darker; it is unlikely that you would want to increase the brightness as it becomes impossible to detect an additional signal in a saturated pixel.

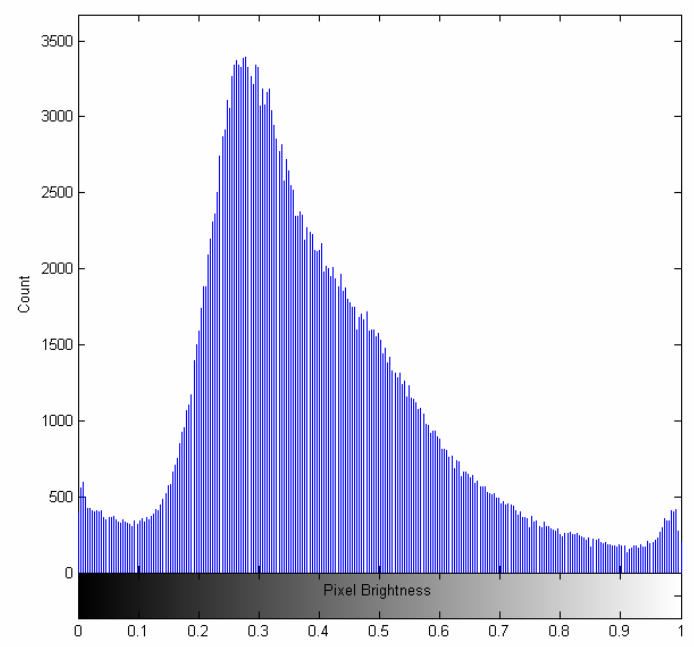

Figure 4: Image Histogram

A sequence of frames was developed by applying the vibration data described in Section 3 in the manner explained in Section 5. An example of the shifting that occurs is shown in Figure 5, where the motion has been greatly magnified for effect. These frame to frame shifts are a major source of noise (background clutter) when trying to detect a weak signal via background subtraction techniques.

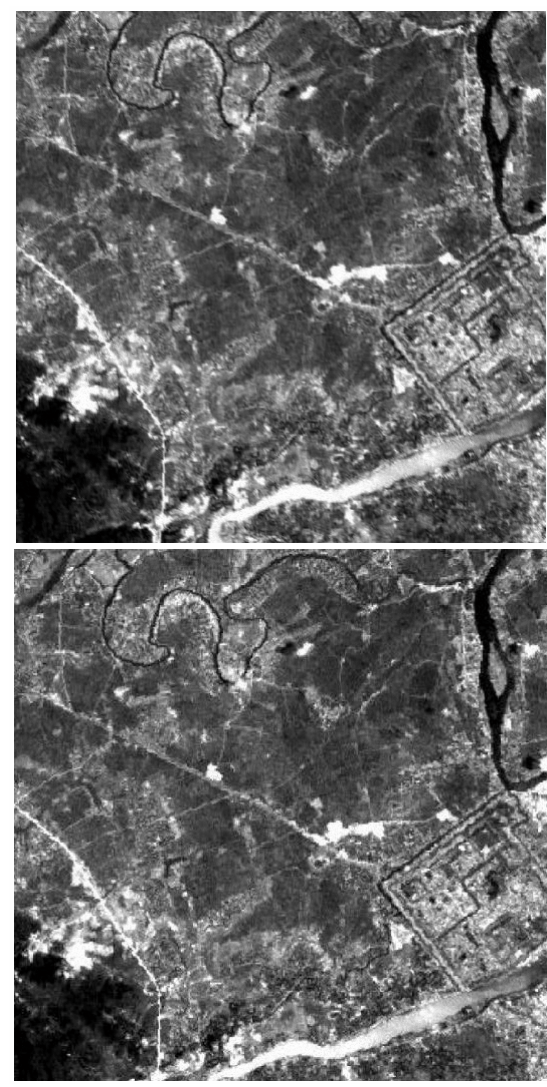

Figure 5: Image Motion Sequence 
Noise was added on a per-pixel, per frame basis as described in Section 5, with the following input parameters:

Read noise: 39 electrons, $1-\sigma$

Pixel non-uniformity: $0.02 \%$

A/D conversion: 8 bits

An example with $5 \mathrm{x}$ the actual noise added is shown in Figure 7, which is a blow-up of the top left portion of the image in Figure 3 (the corresponding noise-free image is shown in Figure 7). Without the 5x amplification in noise, it is very difficult to visually detect the degradation in the image. It should be noted that the dominant noise source for these parameters is signal shot noise.

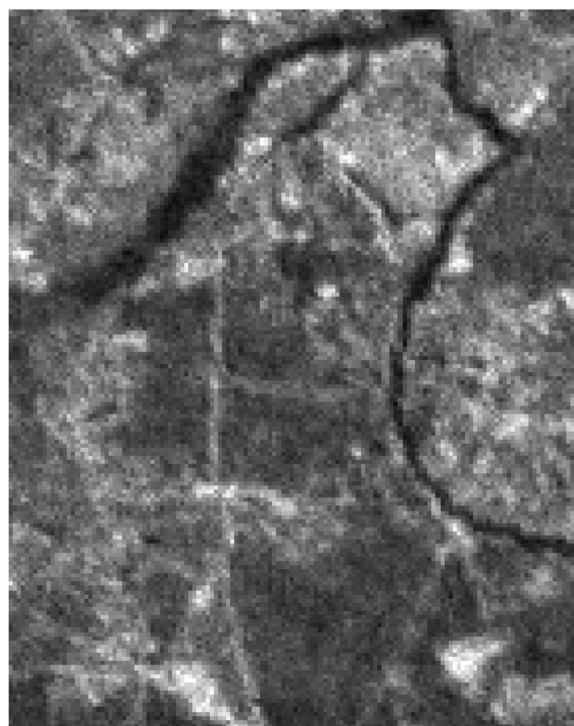

Figure 6: Image with Added Noise

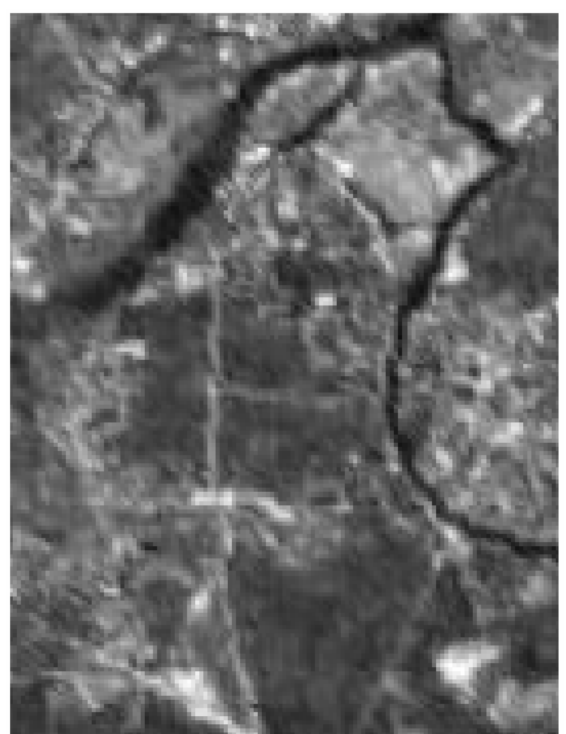

Figure 7: Image Without Added Noise

A sequence of 900 frames (5 seconds at $180 \mathrm{~Hz}$ ) was created by applying the vibration spectra and random noise inputs as described, and then a change file was created by subtracting off the previous image from each successive image. On any given image, the histogram of the remaining noise in the change file looks like Figure 8 , while the standard deviations in the sequence of residual noises are shown in Figure 9; the standard deviation ranged from a minimum of 3.5 to a maximum of 35.8 , with the average standard deviation being 11.6.

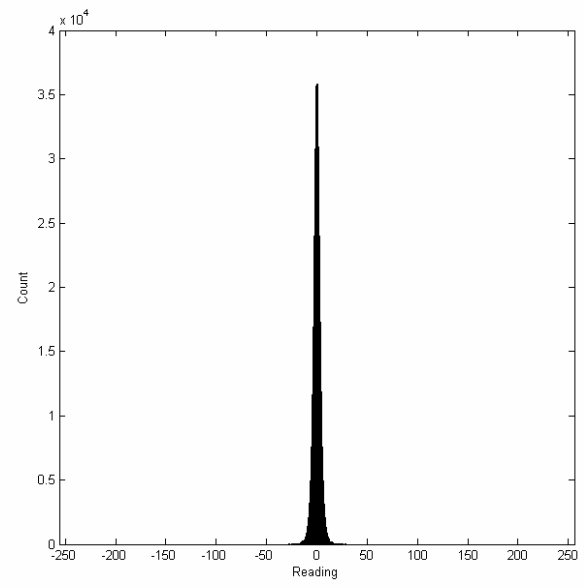

Figure 8: Background Subtracted Histogram

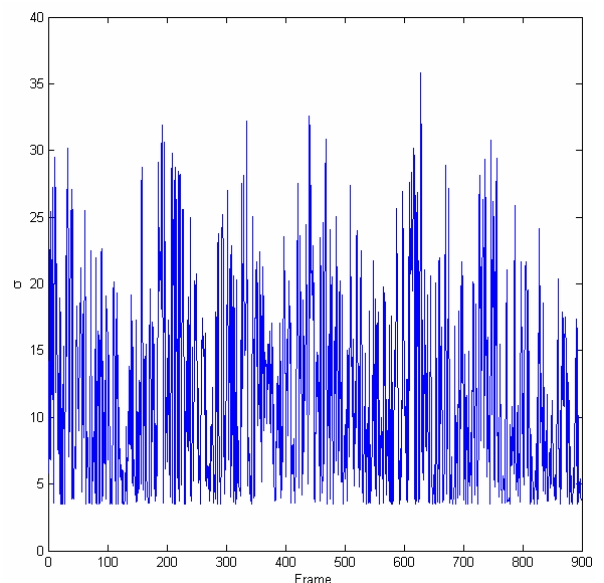

Figure 9: Background Subtracted Noise SD per Frame

Once statistics on the residual noise are known, it then becomes possible to determine the required signal levels to achieve a given false alarm rate and probability of detection. Let us suppose a $1 \%$ false alarm rate over our 5 second window is required. Using Equation (15) gives a per-frame false alarm rate of 1.11E-5. With a simplistic 1-pixel, 1frame detection algorithm, the required per-pixel false alarm rate is 3.9E-11 from Equation (16), which requires a threshold set to a level greater than $6.5 \sigma$. This equates to a threshold at 75 if the mean standard deviation is used and 233 if the maximum value is used. The maximum pixel value per frame is shown in Figure 10; note that a threshold of 75 will produce a plethora of false alarms, while a level of 233 produces none where we would expect to see one only $1 \%$ of the time. Using neither the mean nor the maximum standard deviation has achieved the desired results, so a closer look at what is going on in Figure 9 and Figure 10 is warranted. 


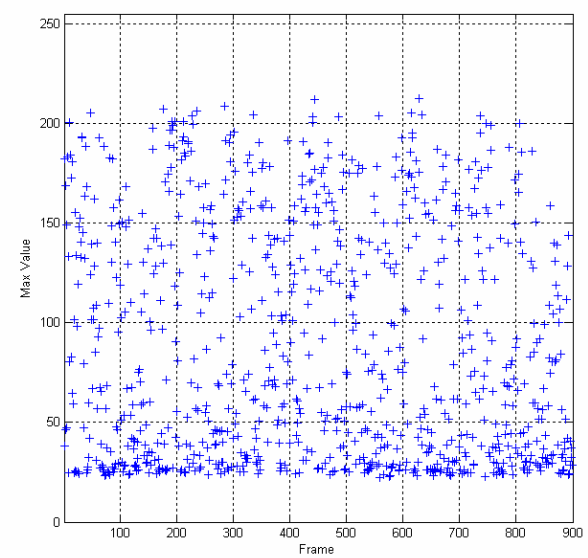

Figure 10: Maximum Change in Pixel Values per Frame

The (cropped) image we are examining contains $531^{2}$ pixels; therefore it should not be surprising that events with probabilities as low as $3.5 \mathrm{E}-6$ occur, which corresponds to almost $4.5 \sigma$. However, Figure 11 shows the number of standard deviations the maximum value is away from the mean in each frame; events are occurring at over $12 \sigma$ away from the mean, which corresponds to a probability of $<1 \mathrm{E}$ 32. Obviously what is going on is that the distribution shown in Figure 8 is not strictly normal in the tails. Examination of where the extremes occur in the image shows that sharp boundaries between high and low valued pixels are the main sources of frame-to-frame variation. In any case, for this particular algorithm, the false-alarm statistics built up in Section 4 can not be used in this manner to produce a valid false alarm rate based threshold. Instead, one would have to take the data shown in Figure 10 and fit it to an extreme value distribution to determine the correct threshold.

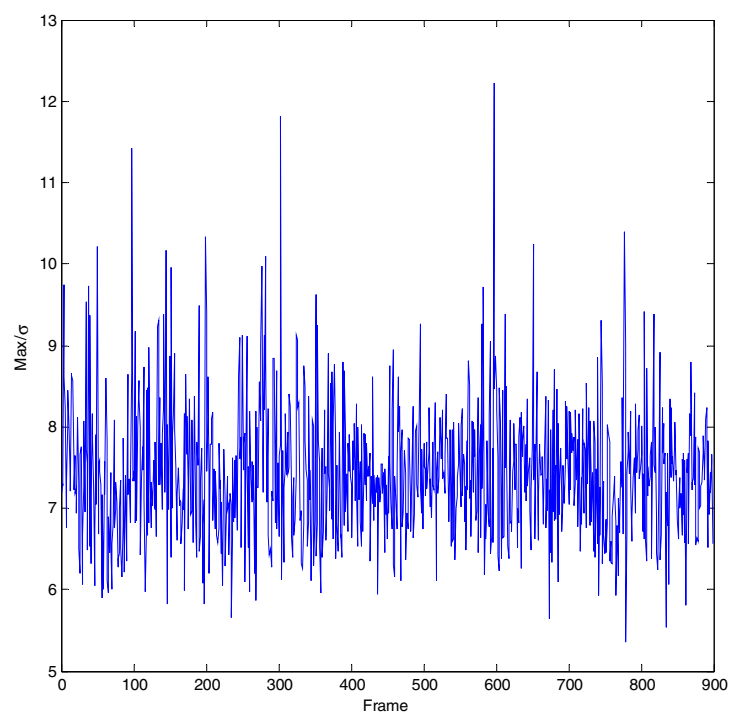

Figure 11: Maximum value/Standard Deviation
The next logical step to take is to require the spot to remain for 2 frames. From Equation (18), this gives a per-pixel false alarm rate of $6.28 \mathrm{E}-6$, which corresponds to a threshold set to $4.4 \sigma$. Changing the detection criteria also changes the residual noise statistics (the $\sigma$ ) from the single frame detection case, in a manner that is dependent on how well the residual noise is correlated from frame to frame and how the background subtraction is performed. For example, if the scheme is $B_{1}{ }^{n}=A^{n}-A^{n-1}, B_{2}{ }^{n}=A^{n}-A^{n-2}$, then the noise in $\mathrm{B}_{2}{ }^{\mathrm{n}}$ will tend to be larger, as more jitter has occurred. If a new frame $\mathrm{C}^{\mathrm{n}}$ is constructed by finding the minimum value for each pixel in $\mathrm{B}_{1}{ }^{\mathrm{n}}, \mathrm{B}_{2}{ }^{\mathrm{n}}$, the standard deviation over the run time becomes as shown in Figure 12, which is only moderately reduced from Figure 9; it has a $\min$ of 3.2 , a max of 33.3 , and an average of 12.11; the maximum values in $\mathrm{C}^{\mathrm{n}}$ are likewise only slightly reduced from those shown in Figure 12. This indicates that this particular scheme produces noise in successive frames that is highly correlated, and the assumptions used to come up with the $4.4 \sigma$ threshold level are suspect (Setting the level to $4.4 \sigma$ when $\sigma$ is the max value of 33.3 gives a threshold at 147 ; this produces 100 false alarms during this period!).

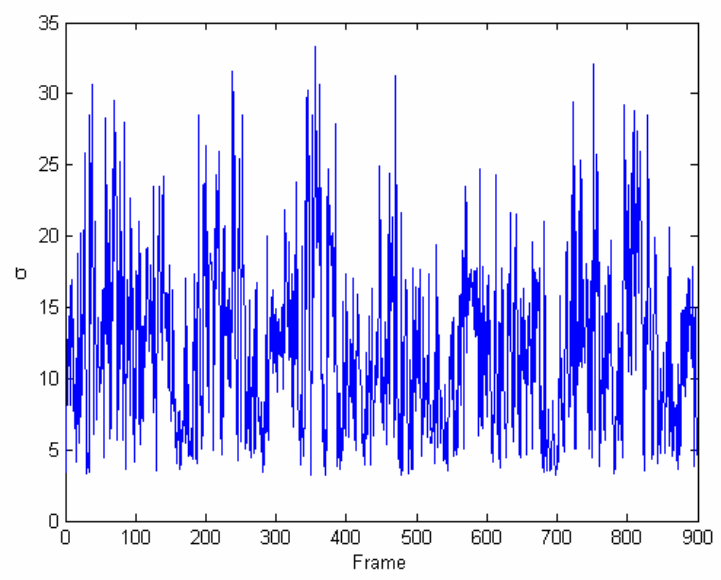

Figure 12: Two Frame Noise SD per Frame

An alternate scheme to achieve the desired two frames false alarm rates is to let $\mathrm{B}_{1}{ }^{\mathrm{n}}=\mathrm{A}^{\mathrm{n}}-\mathrm{A}^{\mathrm{n}-1}, \mathrm{~B}_{2}{ }^{\mathrm{n}}=\mathrm{A}^{\mathrm{n}-1}-\mathrm{A}^{\mathrm{n}-2}$. In this case, the noise becomes much less correlated and the standard deviations are reduced to the values shown in Figure 13, with a min of 2.7, a max of 22.8, and an average value of 9.8. The maximum pixel value that is present in both frames is shown in Figure 14; it is clear that a much lower threshold can be tolerated in this case. 


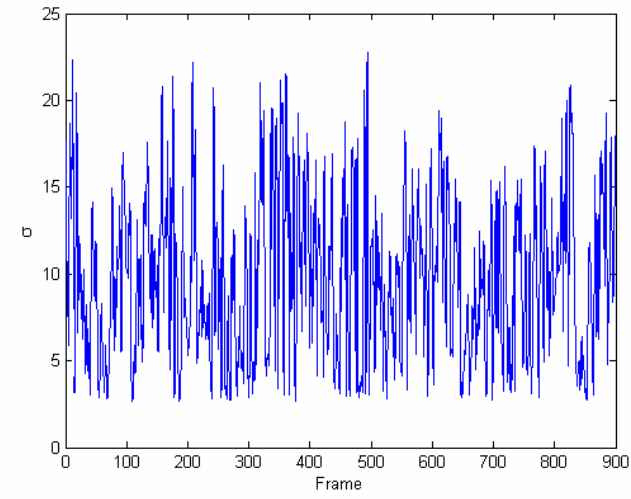

Figure 13: Alternate Two Frame SD

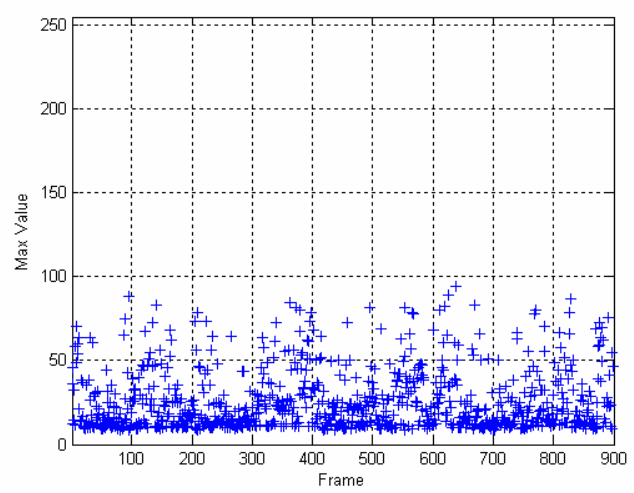

Figure 14: Alternate Two Frame Max Change per Frame

Setting the threshold at $4.4 \sigma$, and using the maximum $\sigma$ of 22.8 , gives a level of 97; the maximum value of the noise does not exceed this level in any of the frames.

Finally, combing the multi-frame criteria shown above with the pair of pixels criteria as described in Equations 18-19 reduces the observed maximum values even more. The results for this simulation are shown in

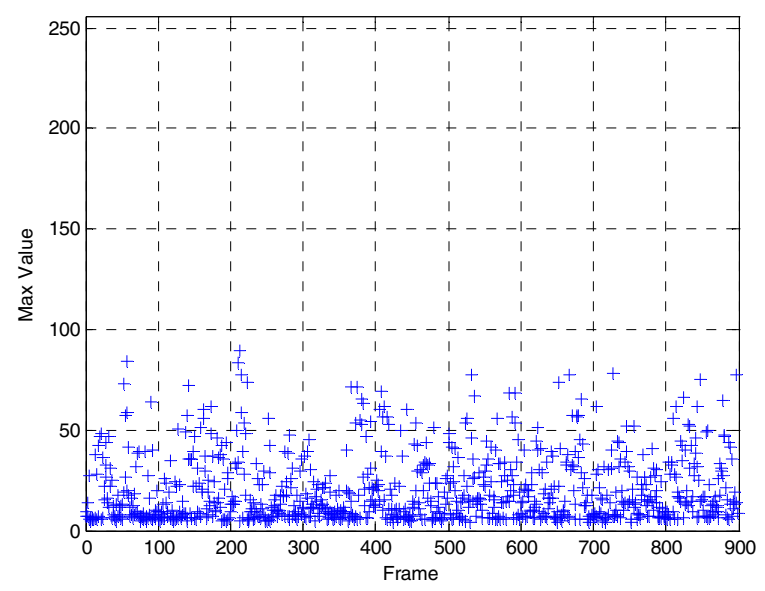

Figure

15

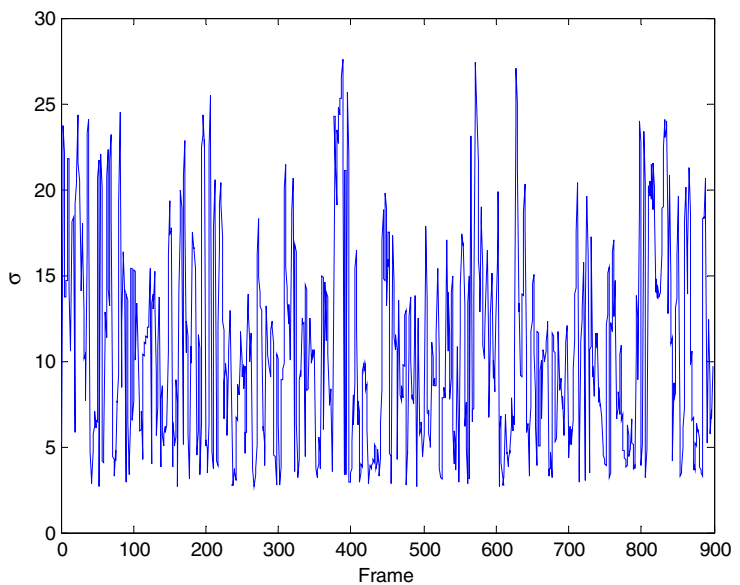

Figure 16. Note that the maximum values have been reduced by about $10 \%$ from the previous case considered. The per-pixel false alarm rate has been dropped all the way to $1.8 \mathrm{E}-3$, which corresponds to a threshold set to $2.9 \sigma$. The standard deviation for this process, shown in

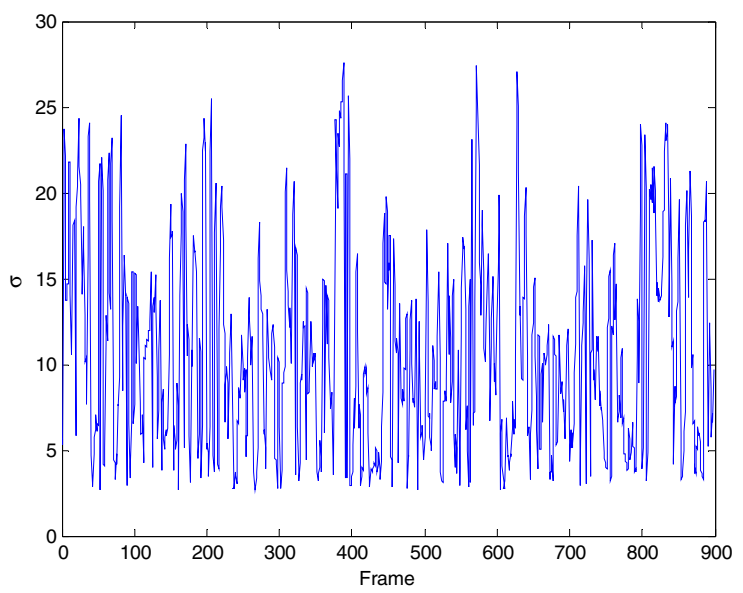

Figure 16, has a mean value of 10.8 and a maximum of 27.6. As the threshold has been set at $2.9 \sigma$, the non-normal behavior in the tails of the distribution is not apparent, and we are much closer to what is expected: At a threshold of $2.9 \sigma$ where the maximum sigma is used, we get 5 instances of threshold exceedance, which is more than we would expect. 


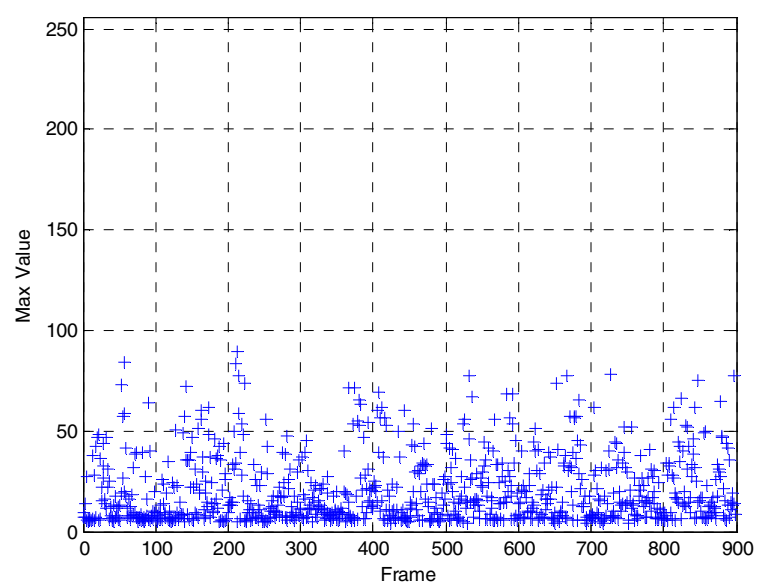

Figure 15: Two Frame, Two Pixel Max Values

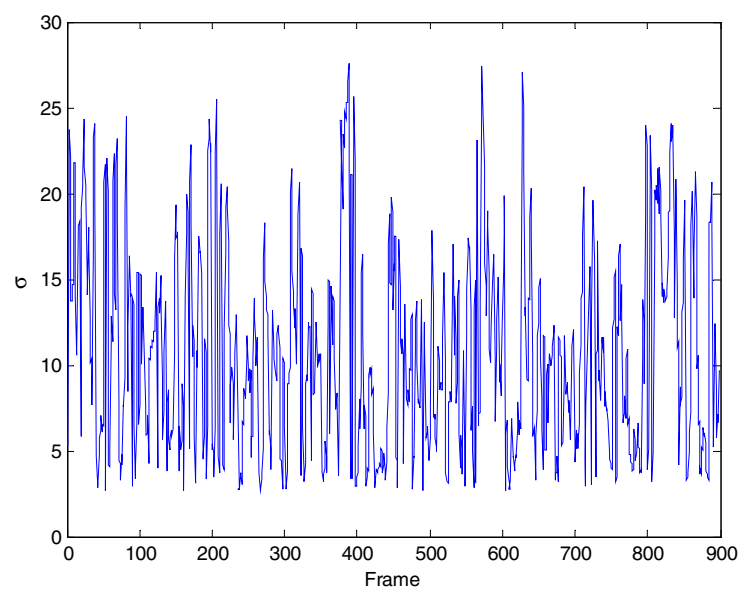

Figure 16: Two Frame, Two Pixel SD

Table 2 summarizes the results from this section. The first column lists each algorithm that was tested, along with the required per-pixel false alarm rates. Type A corresponds to the single-frame algorithm, Type B the first two-frame algorithm, Type $\mathrm{C}$ the second two-frame algorithm, and Type D the two-frame, two-pixel algorithm. The second column indicates the estimated threshold level to achieve the desired false alarm rate based on the mean standard deviations, while the third column is based on the maximum recorded standard deviation. The final column represents the maximum value recorded in each simulation.

\begin{tabular}{|lrrr|}
\hline \multicolumn{4}{|c|}{ Threshold Setting Results } \\
\hline \multicolumn{4}{|c|}{ Threshold levels (1\% FAR) } \\
Type, PPFAR & Est (mean) & Est (max) & Actual \\
A / 3.9E-11 & 75 & 233 & 231 \\
B / 6.28E-6 & 53 & 147 & 208 \\
C / 6.28E-6 & 43 & 100 & 101 \\
D / 1.8E-3 & 31 & 80 & 88 \\
\hline
\end{tabular}

Table 2: Threshold Setting Results

\section{Conclusions}

An acquisition detection simulation was developed for detecting faint point source-like signals against bright and highly cluttered background environments. The simulation incorporated electronic and other inherent noise sources, and accounted for vibration-induced image jitter. Detection statistics were developed for several different detection algorithms, and an example using a representative Landsat image was presented to demonstrate the effectiveness of the simulation and the derived detection statistics.

The simulation found that observed maximum threshold values fell as expected with decreasing per-pixel false alarm rate requirements as more restrictive detection algorithms were used. However, it was found that the equations developed in Section 4 did not provide an absolute quantitative match to determining the expected required threshold levels. This was found to be primarily due to nonnormal behavior in the tails of the distributions, caused by correlations in bright and dark pixels.

Future work will involve modifying the simulation for tracking applications as well as detecting multiple sources when very bright sources are present. Further development of the detection statistics to more accurately match the observed results will also be performed.

\section{REFERENCES}

[1] G. Griseri, 'Silex Mission Overview', presented at the International Workshop on Control of Optical Systems Conference, Breckenridge, CO Feb. 2003.

[2] G. Fletcher, T Hicks, B. Laurent, 'The SILEX Optical Interorbit Link Experiment', Electronics \& Communications Engineering Journal, Dec. 1991.

[3] K. Nakagawa, A. Yamamoto, M. Toyoda, 'Performance test result of LUCE (laser utilizing communications equipment) engineering model', Proc. SPIE Vol. 3932, Free-Space Laser Communication Technologies XII, 2000.

[4] Isaac I. Kim, Brian Riley, Nicholas M. Wong, Mary Mitchell, Wesley Brown, Harel Hakakha, Prasanna Adhikari, and Eric J. Korevaar, 'Lessons learned for STRV2 satellite-to-ground lasercom experiment', Proc. SPIE Int. Soc. Opt. Eng. 4272, No 1, 2001

[5] G. Baister, P. Gatenby, J. Lewis and M. Wittig, 'The SOUT Optical Intersatellite Communication Terminal', IEE Proceedings on Optoelectronics, Vol. 141, No. 6, Dec. 1994.

[6] M. McCarter, 'Satellite Transformation', Military Information Technology, Vol. 9, No. 4, June 13, 2005. 
[7] S. Lambert and W. Casey, Laser Communications in Space, Artech House, Boston, 1995.

[8] M. Guelman, A. Kogan, A. Kazarian, A. Livne, M. Orenstein, H. Michailik, and S. Arnon, 'Acquisition and Pointing Control for Inter-Satellite Laser Communications', IEEE Transactions on Aerospace and Electronic Systems, Vol. 40, No 4, October 2004.

[9] M. V. Newberry, 'Signal-to-Noise Considerations for Sky-Subtracted CCD Data', Publications of the Astronomical Society of the Pacific, Vol. 102, January 1991.

[10] G. Ortiz, S. Lee and H. Hemmati, 'Technology Maturation for Multi-Gigabit/sec Optical Communications Transceiver for Earth Science', NASA's Earth Science and Technology Conference, Pasadena, California, 2002.

[11] R.D. Nelson, T.H. Ebben, R.G. Marshalek, 'Expreimental Verification of the Pointing Error Distribution of an Optical Intersatellite Link', SPIE Free Space Laser Communication Technologies, Vol 885, 1998.

[12] M. Wittig, L. van Holtz, D.E.L. Tunbridge, and H.C. Vermeulen, 'In-Orbit Measurements of Microaccelerations of ESA's Communication Satellite OLYMPUS', SPIE FreeSpace Laser Communication Technologies II, Vol. 1218, 1990.

[13] H. Kingston, Detection of Optical and Infrared Radiation, Springer-Verlag, Berlin, 1978.

[14] C. W. Helstrom, 'The Detection and Resolution of Optical Signals', IEEE Transactions on Information Theory, Oct 1964.

\section{[15] http://landsat7.usgs.gov/}

[16] Hardie, R.C., Hayat, M.M., Armstrong, E., and Yasuda, B., 'Scene-Based Nonuniformity Correction with Video Sequences and Registration', Applied Optic Vol. 39, No. 8, March 10, 2000.

\section{BIOGRAPHY}

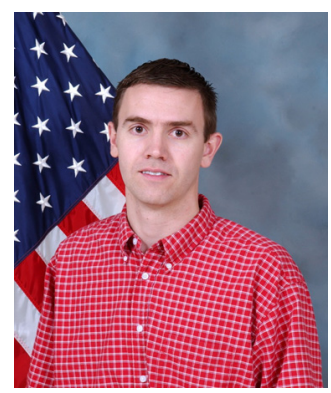

Dr Charles "Bill" Hindman received his BS degree from the University of Idaho in Mechanical Engineering, his MS degree from the University of Arizona in Mechanical Engineering, and his Ph.D. degree from the University of Colorado at Boulder in Aerospace Engineering. Currently he is the Pointing, Acquisition and Tracking and Laboratory Technical
Leads for the multi-user laser communications programs at the Air Force Research Laboratory. His research interests include the modeling, simulation and testing of laser propagation and detection, multi-target tracking, and control systems.

Dr Seth Lacy is a Research Aerospace Engineer at the Air

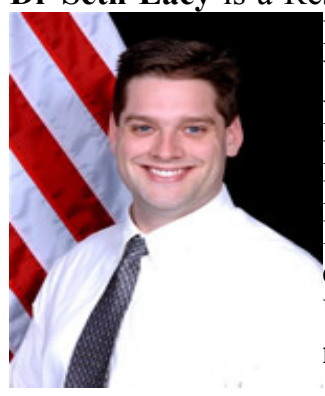
Force Research Laboratory's Space Vehicles Directorate, Kirtland AFB NM. He received B.S. degrees in Physics and Aeronautical Engineering from the California Institute of Technology in 1997. He continued his education at the University of Michigan where he received the M.S. degree in Aerospace Engineering in 1999, the M.S. degree in Mathematics and his Ph.D. in Aerospace Engineering in 2002. He has published articles in refereed conferences and journals on various aspects of system identification and control. He is currently developing laser communications technology.

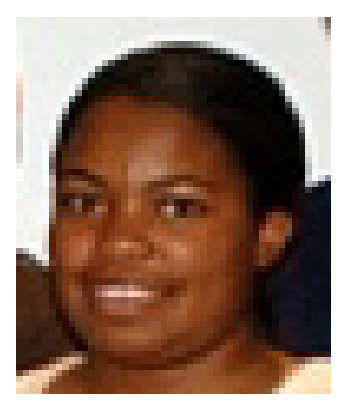

Nicole Hatten is currently a graduate student at Mercer University in Macon, GA pursuing a Master's of Science degree in Electrical Engineering. She received her BS at Wilberforce University in December of 2003. Her Master's work specializes in feedback control systems with applications to robotics. Ms Hatten was a 2005 space scholar at the Air Force Research Laboratory, Space Vehicles Directorate, performing research on image processing algorithms for laser communications systems. 